\title{
THEORIE DES VALEURS EXTREMES ET LA TARIFICATION DE L'EXCESS OF LOSS
}

\author{
L. D'HoOGE
}

Bruxelles, Belgique

I. NOTATIONS

Soit $x_{1} \leqslant x_{2} \leqslant \ldots \leqslant x_{n-m+1} \leqslant \ldots \leqslant x_{n-p+1} \leqslant \ldots \leqslant x_{n}$ un échantillon ordonné de taille $n$ d'une distribution dont $F(x)$ et $T(x)$ sont respectivement la fonction de répartition et la fonction de densité.

Nous appelons $x_{n-m+1}$ la valeur de rang $m$ de l'échantillon $\left(x_{1}\right.$, $x_{2}, \ldots x_{n}$ ).

Pour simplifier l'écriture nous remplacerons partout l'indice $n-m+$ I par $m$.

Avec cette convention nous notons la valeur caractéristique de rang $m$ et l'intensité de rang $m$ respectivement par $u_{m}$ et $\alpha_{m}[\mathrm{r}]$. Par définition on a:

et

$$
\begin{aligned}
F\left(u_{m}\right) & =\mathrm{I}-\frac{m}{n} \\
\alpha_{m} & =\frac{n}{m} T\left(u_{m}\right)
\end{aligned}
$$

Notons enfin par $F_{m}(x)$ et $T_{m}(x)$ la fonction de répartition et la fonction de densité de $x_{m}$ pour $n \rightarrow \infty$ et $m$ fixe.

\section{INTRODUCTION}

2.1. Avant de résumer les différents points traités dans cette note, rappelons les particularités qui caractérisent la distribution asymptotique de la plus grande valeur $x_{1}$ d'un échantillon. On sait que cette distribution existe si et seulement si la fonction de répartition $F(x)$ appartient à un des trois types suivants [2]:

1) Nous avons adopté les notations de E. J. Gumbel: „Statistics of Extremes".

2) Gnedenko: „Sur la distribution limite du terme maximum d'une série aleatoire" (Ann. Math. Stats 44). 
a) Type I: $F(x)$ est du type exponentiel.

Le domaine de $x$ est illimité vers la droite et $F(x)$ tend vers I pour $x \rightarrow+\infty$ au moins aussi rapidement qu'une fonction exponentielle.

Tous les moments de $F(x)$ existent.

b) Type II:F(x) est du type de Cauchy.

Le domaine de $x$ est illimité vers la droite.

$F(x)$ est caractérisé par deux paramètres:

$\varepsilon$ étant la borne inférieure de $x(\varepsilon \gtrless 0)$

et

$$
k \text { défini } \underset{x \rightarrow \infty}{\operatorname{par} \lim } \frac{\mathrm{I}-F(x)}{\mathrm{I}-F(c x)}=c^{k}(\mathrm{c}>0, k>0)
$$

(condition de Gnedenko).

$F(x)$ ne possède pas de moments d'ordre supérieur ou égal à $k$.

c) Type III: $F(x)$ est du type borné.

Le domaine de $x$ est limité vers la droite par $x=w$ (premier paramètre). On a $F(w)=$ I et $F(w-\eta)<$ I $(\eta>0)$.

$F(x)$ est caractérisé par un deuxième paramètre $l$ défini par $\lim _{x \rightarrow \infty} \frac{\mathrm{I}-F(c x+w)}{\mathrm{I}-F(x+w)}=c^{l}(c>0, l>0)$ (condition de Gnedenko).

\subsection{Résumé.}

a) Dans le chapitre 3 nous espérons donner une réponse à une question posée dans : „The Astin Bulletin”, Vol. II, Part III, page 3I4 (avril 1963) à savoir: "to develop the formal derivation for the (asymptotic) distribution of $m$ ' th values in terms of the largest value".

Nous établirons pour les trois types de fonctions une expression de $T_{m}(x)$ en fonction des paramètres extrêmes $u_{1}$ et $\alpha_{1}$.

b) En appliquant la méthode de Beard [I] et grâce aux résultats obtenus au chapitre 3 on établira dans le chapitre 4 des expressions simples permettant le calcul de $E(S)$ pour les différents types de fonctions $F(x)$.

1) R. E. BEARD: „Statistical Theory of extreme values, and an application to excess of loss insurance". 
$F(x)$ étant la fonction de répartition des sinistres survenus; ( $x$ étant la somme payée).

$E(S)$ étant l'espérance mathématique de la somme $S$ payée par le réassureur pendant une période de temps de référence déterminée (réassurance excess of loss).

On montrera en conclusion de ce chapitre que la méthode suivie revient, comme il fallait s'y attendre, à admettre pour le ,tail" de $F(x)$ une fonction bien spécifiée, dont on estime les paramètres à partir d'un échantillon donné.

c) Dans le chapitre 5 enfin on établira une expression plus générale de $E(S)$ valable pour une loi de survenance des sinistres quelconque.

3. LA FONCTION DE DENSITÉ $T_{m}(x)$ EN FONCTION DES PARAMÈTRES EXTRÊMES $u_{1}$ ET $\alpha_{1}$

\subsection{Théorème $A$.}

Si $F(x)$ est du type I, alors pour:

$$
n \rightarrow \infty
$$

$m$ et $p$ fixes (et donc petits par rapport à $n$ ), on a l'identité suivante en $x$ :

$$
\alpha_{m}\left(x-u_{m}\right)=\alpha_{p}\left(x-u_{p}\right)+\lg m-\lg p
$$

(lg étant le logarithme népérien).

\subsubsection{Remarque préliminaire.}

Si $F(x)$ est une loi exponentielle la relation (3.I) est satisfaite pour toute valeur de $n$.

En effet, pour $F(x)=\mathrm{I}-e^{-\lambda x}$ et donc $T(x)=\lambda e^{-\lambda x}$ on vérifie facilement que:

$$
\begin{aligned}
& u_{p}=\frac{\mathrm{I}}{\lambda}(\lg n-\lg p) \\
& u_{m}=\frac{\mathrm{I}}{\lambda}(\lg n-\lg m) \\
& \alpha_{p}=\lambda \\
& \alpha_{m}=\lambda
\end{aligned}
$$

Dès lors, la relation (3.I) est vérifiée quelle que soit la valeur de $n$. 
On peut donc s'attendre à ce que cette relation soit vérifiée asymptotiquement pour toute loi $F(x)$ du type exponentiel.

3.1.2. Démonstration du théorème $A$.

On peut écrire l'identité:

$$
\alpha_{m}\left(x-u_{m}\right)=\frac{\alpha_{m}}{\alpha_{p}} \alpha_{p}\left(x-u_{p}\right)+\alpha_{m}\left(u_{p}-u_{m}\right)
$$

a) Démontrons que:

$$
\lim _{n \rightarrow \infty} \alpha_{m}\left(u_{p}-u_{m}\right)=\lg m-\lg p
$$

En développant $F(x)$ en série de Taylor à partir de la valeur caractéristique $u_{m}$ on a pour $u_{m}$ grand et $x$ dans le voisinage de $u_{m}[\mathrm{I}]$ :

$$
F(x)=\mathrm{I}-\frac{m}{n} e^{-\alpha_{m}\left(x-u_{m}\right)}
$$

Pour $x=u_{p}$ et en vertu de $F\left(u_{p}\right)=\mathrm{I}-\frac{p}{n}$ la relation (3.4) devient:

$$
p=m e^{-\alpha_{m}\left(u_{p}-u_{m}\right)}
$$

d'où on déduit immédiatement la relation à démontrer (3.3).

b) Démontrons ensuite que:

$$
\lim _{n \rightarrow \infty} \frac{\alpha_{m}}{\alpha_{p}}=\mathrm{I}
$$

En développant $F(x)$ en série de Taylor à partir de la valeur caractéristique $u_{p}$ on a pour $u_{p}$ grand et $x$ dans le voisinage de $u_{p}$ :

$$
F(x)=\mathrm{I}-\frac{p}{n} e^{-\alpha_{p}\left(x-u_{p}\right)}
$$

Pour $x=u_{m}$ et en vertu de $F\left(u_{m}\right)=\mathrm{I}-\frac{m}{n}(3 \cdot 7)$ devient:

$$
m=p e^{-\alpha_{p}\left(u_{m}-u_{p}\right)}
$$

Des relations (3.5) et (3.8) on déduit:

$$
\alpha_{m}\left(u_{p}-u_{m}\right)\left(\frac{\alpha_{p}}{\alpha_{m}}-\mathrm{I}\right)=0
$$

1) E. J. Gumbel: „Statistics of Extremes” (page I68). 
et puisque pour $m \neq p$ on a $\alpha_{m}\left(u_{p}-u_{m}\right) \neq 0$ il faut nécessairement que $\frac{\alpha_{p}}{\alpha_{m}}=\mathrm{I}$.

c) la substitution des relations (3.3) et (3.6) en (3.2) démontre le théorème.

\subsubsection{Cas particulier.}

Pour $p=\mathrm{I}$ la relation (3.I) devient:

$$
\alpha_{m}\left(x-u_{m}\right)=\alpha_{1}\left(x-u_{1}\right)+\lg m
$$

Cette relation permet donc d'exprimer la variable $\alpha_{m}\left(x-u_{m}\right)$ en fonction du rang $m$ et des paramètres extrêmes $\alpha_{1}$ et $u_{1}$.

3.1.4. $T_{m}(x)$ en fonction des paramètres extrêmes $u_{1}$ et $\alpha_{1}$.

Rappelons que $T_{m}(x)$ est la fonction de densité de $x_{m}$ pour $n \rightarrow \infty$ et $m$ fixe.

Si $m$ est petit par rapport à $n$ on sait que $[\mathrm{I}]$ :

$T_{m}(x)=\alpha_{m} \frac{m^{m}}{(m-\mathrm{I}) !} \exp \left[-m \alpha_{m}\left(x-u_{m}\right)-m e^{-\alpha_{m}\left(x-u_{m}\right)}\right]$ (3.I0)

Dans cette expression de $T_{m}(x)$ les paramètres $\alpha_{m}$ et $u_{m}$ dépendent de $F(x)$ et du rang $m$.

Il en résulte que les expressions de $T_{m}(x)$ ne sont connues analytiquement que pour autant qu'on puisse déterminer pour chaque valeur de $m$ les paramètres correspondants $\alpha_{m}$ et $u_{m}$.

Si $F(x)$ n'est pas connu (ce qui est souvent le cas en pratique), mais si on sait que $F(x)$ est du type exponentiel et si en plus on connaît les paramètres extrêmes $\alpha_{1}$ et $u_{1}$, on peut appliquer (3.9) et dès lors (3.ro) devient:

$$
T_{m}(x)=\alpha_{1} \frac{\mathrm{I}}{(m-\mathrm{I}) !} \exp \left[-m \alpha_{1}\left(x-u_{1}\right)-e^{-\alpha_{1}\left(x-u_{1}\right)}\right]
$$

Dans cette expression de $T_{m}(x)$ interviennent le rang $m$ et uniquement les paramètres extrêmes $\alpha_{1}$ et $u_{1}$.

1) E. J. Gumbel: „Statistics of Extremes” (page 187). 
Pour $\mathrm{m}=\mathrm{I}$ (3.II) devient:

$$
T_{1}(x)=\alpha_{1} \exp \left[-\alpha_{1}\left(x-u_{1}\right)-e^{-\alpha_{1}\left(x-u_{1}\right)}\right]
$$

et on retrouve l'expression connue de la fonction de densité de la plus grande valeur $x_{1}$.

\subsection{Théorème $B$.}

On a pour:

$$
n \rightarrow \infty
$$

$m$ et $p$ fixes (et donc petits par rapport à $n$ ):

$$
u_{m}-\varepsilon=\left(u_{p}-\varepsilon\right)\left(\frac{p}{m}\right)^{1 / k}
$$

si $F(x)$ est du type II

et

$$
w-u_{m}=\left(w-u_{p}\right)\left(\frac{m}{p}\right)^{1 / l}
$$

si $F(x)$ est du type III.

$(\varepsilon, k, w, l$ sont les paramètres définis en $2 . \mathrm{I})$.

3.2.1. Démonstration du théorème $B$.

On a pour $u_{m}$ grand et $x$ dans le voisinage de $u_{m}$ :

$$
F(x)=\mathrm{I}-\frac{m}{n}\left(\frac{u_{m}-\varepsilon}{x-\varepsilon}\right)^{k}
$$

si $F(x)$ est du type II.

Par contre, si $F(x)$ est du type III, on a pour $u_{m}$ proche de $w$ et $x$ dans le voisinage de $u_{m}$ :

$$
F(x)=\mathbf{I}-\frac{m}{n}\left(\frac{w-x}{w-u_{m}}\right)^{l}
$$

Pour $x=u_{p}$ et en vertu $\operatorname{de} F\left(u_{p}\right)=\mathrm{I}-\frac{p}{n}$ les relations (3.14) et (3.I5) prouvent immédiatement le théorème $B$.

3.2.2. Cas particuliers.

Pour $p=\mathrm{I}$ les relations (3.12) et (3.13) deviennent: 


$$
u_{m}-\varepsilon=\left(u_{1}-\varepsilon\right)\left(\frac{\mathrm{I}}{m}\right)^{1 / k}
$$

$(F(x)$ du type II).

et

$$
w-u_{m}=\left(w-u_{1}\right) m^{1 / l}
$$

$(F(x)$ du type III).

Ces deux relations permettent d'exprimer la valeur caractéristique de rang $m$ en fonction du rang $m$, de la valeur caractéristique extrême $u_{1}$, et des paramètres $k, \varepsilon(w, l)$ indépendants du rang $m$.

\subsection{3. $T_{m}(x)$ en fonction $d u$ paramètre extrême $u_{1}$.}

Si $m$ est petit par rapport à $n$ on sait que:

$$
T_{m}(x)=\frac{k}{u_{m}-\Sigma} \cdot \frac{m^{m}}{(m-\mathrm{I}) !}\left(\frac{u_{m}-\varepsilon}{x-\varepsilon}\right)^{k m+1} e^{-m\left(\frac{u_{m}-\varepsilon}{x-\varepsilon}\right)^{k}}
$$

si $F(x)$ est du type II

$$
\text { et } T_{m}(x)=\frac{l}{w-u_{m}} \cdot \frac{m^{m}}{(m-\mathrm{I}) !}\left(\frac{w-x}{w-u_{m}}\right)^{l m-1} \mathrm{e}^{-m\left(\frac{w-x}{w-u_{m}}\right)^{l}}
$$

si $F(x)$ est du type III.

Dans ces expressions de $T_{m}(x)$ interviennent d'une part les paramètres $k, \varepsilon(l, w)$ dépendant uniquement des propriétés de $F(x)$ et d'autre part le paramètre $u_{m}$ dépendant de $F(x)$ et du rang $m$.

En appliquant (3.16) et (3.17) les expressions de $T_{m}(x)$ (3.18) et (3.I9) deviennent:

$$
T_{m}(x)=\frac{k}{u_{1}-\varepsilon} \cdot \frac{\mathrm{I}}{(m-\mathrm{I}) !}\left(\frac{u_{1}-\varepsilon}{x-\varepsilon}\right)^{k m+1} e^{-\left(\frac{u_{1}-\varepsilon}{x-\varepsilon}\right)^{k}}
$$

si $F(x)$ est du type II

et $\quad T_{m}(x)=\frac{l}{w-u_{1}} \frac{\mathrm{I}}{(m-\mathrm{I}) !}\left(\frac{w-x}{w-u_{1}}\right)^{l m-1} e^{-\left(\frac{w-x}{w-u_{1}}\right)^{l}}$

si $F(x)$ est du type III. 
Dans ces expressions apparaissent, à part le rang $m$ et les paramètres $k, \varepsilon(l, w)$, uniquement la valeur caractéristique extrême $u_{1}$.

Pour $m=\mathrm{I}(3.20)$ et (3.2I) deviennent:

$$
T_{1}(x)=\frac{k}{u_{1}-\varepsilon}\left(\frac{u_{1}-\varepsilon}{x-\varepsilon}\right)^{k+1} e^{-\left(\frac{u_{1}-\varepsilon}{x-\varepsilon}\right)^{k}}
$$

si $F(x)$ est du type II

$$
\text { et } T_{1}(x)=\frac{\mathrm{I}}{w-u_{1}}\left(\frac{w-x}{w-u_{1}}\right)^{l-1} e^{-\left(\frac{w-x}{w-u_{1}}\right)^{l}}
$$

si $F(x)$ est du type III.

On retrouve ainsi les expressions connues des fonctions de densité de la plus grande valeur $x_{1}$.

En conclusion de ce chapitre rappelons que généralement les expressions de $T_{m}(x)$ données par (3.I0), (3.II), (3.I8), (3.I9), (3.20) et (3.2I) ne sont valables que pour autant que $m$ soit petit par rapport à $n$.

\section{APPLICATION DE LA THÉORIE DES VALEURS EXTRÊMES À LA RÉASSURANCE EXCESS OF LOSS}

\subsection{Position du probleme.}

Dans une catégorie de risques déterminés le réassureur prend à sa charge, la partie de chaque sinistre qui dépasse la valeur $L$ (excess-level).

Appelons $S$ la somme totale payée par le réassureur pendant une période de temps de référence déterminée.

Noús nous proposons de calculer pour la période de temps de référence l'espérance mathématique $E(S)_{a}$ de la somme totale $S$ payée par le réassureur dans les hypothèses suivantes:

a) La fonction de répartition $F(x)$ des sinistres survenus ( $x$ étant la somme payée) n'est pas connue, mais ne subit aucune modification dans le temps.

b) On a observé $N$ séries indépendantes consécutives chacune de n sinistres indépendants ( $n$ et $N$ suffisamment grand).

Toutefois comme matériel statistique nous disposons uniquement pour chaque série considérée du montant du sinistre le plus élevé.

Soit $x_{1}^{(1)}, x_{1}^{(2)} \ldots x_{1}^{(N)}$ les montants des $N$ plus grands sinistres. 


\subsection{Remarque préliminaire.}

En admettant que $F(x)$ appartienne à un des trois types de fonctions mentionnés au chapitre 2, l'étude de l'échantillon $\left(x_{1}{ }^{(1)}, x_{1}{ }^{(2)} \ldots x_{1}{ }^{(N)}\right)$ nous permettra généralement de décider à quel type de fonction $F(x)$ appartient et d'estimer par l'un ou l'autre procédé les paramètres:

$\alpha_{1}$ et $u_{1}$ si $F(x)$ est du type I

$k, \varepsilon$ et $u_{1}$ si $F(x)$ est du type II

$l$, w et $u_{1}$ si $F(x)$ est du type III.

(cfr Statistics of Extremes - E. J. Gumbel).

4.3. Calcul de $E(S)_{a}$ par la méthode de Beard.

Dans sa note ,Statistical theory of extreme values and an application to excess of loss reinsurance", Beard trouve le résultat suivant:

$$
E(S)_{a}=\frac{I}{\alpha_{1}} e^{-\alpha_{1}\left(L-u_{1}\right)}
$$

Dans cette expression de $E(S)_{a}$ interviennent uniquement $L$ et les paramètres $\alpha_{1}$ et $u_{1}$ estimés à partir de l'échantillon $\left(x_{1}^{(1)}, x_{1}^{(2)} \ldots . x_{1}^{(N)}\right)$.

Les résultats obtenus au chapitre 3 de cette note nous permettent de confirmer ce résultat pour toute loi $F(x)$ du type $I$ et de trouver des expressions simples de $E(S)_{a}$ dans les cas où $F(x)$ serait du type II ou du type III.

4.3.1. $F(x)$ est du type $I$.

On peut écrire:

$$
E(S)_{a}=\int_{\Sigma}^{+\infty}(t-L) P(t) d t
$$

où $P(t) d t$ est la somme des probabilités que les variables $x_{1}, x_{2}$, $\ldots x_{m}, \ldots$ prennent une valeur comprise entre $t$ et $t+d t$.

Cette somme est donnée par:

$$
P(t) d t=\sum_{m=1}^{\infty} T_{m}(t) d t
$$


Comme $F(x)$ est du type exponentiel on peut exprimer $T_{m}($ par (3.II) et dès lors (4.I) devient:

$$
P(t) d t=\sum_{m=1}^{\infty} \alpha_{1} \frac{\mathrm{I}}{(m-\mathrm{I}) !} \exp \left[-m \alpha_{1}\left(t-u_{1}\right)-e^{-\alpha_{1}\left(t-u_{1}\right)}\right] d t .
$$

Cette dernière expression se réduit à:

$$
P(t) d t=\alpha_{1} e^{-\alpha_{1}\left(t-u_{1}\right)} d t
$$

Dès lors:

$$
E(S)_{a}=\int_{L}^{+\infty}(t-L) \alpha_{1} e^{-\alpha_{1}\left(t-u_{1}\right)} d t
$$

Après calculs on trouve:

$$
E(S)_{a}=\frac{\mathrm{I}}{\alpha_{1}} e^{-\alpha_{1}\left(L-u_{1}\right)}
$$

4.3.2. $F(x)$ est du type $I I$.

Dans ce cas en appliquant (3.20), (4.I) devient:

$$
P(t) d t=\sum_{m=1}^{\infty}\left(\frac{k}{u_{1}-\varepsilon}\right) \cdot \frac{\mathrm{I}}{(m-\mathrm{I}) !}\left(\frac{u_{1}-\varepsilon}{t-\varepsilon}\right)^{k m+1} e^{-\left(\frac{u_{1}-\varepsilon}{t-\varepsilon}\right)^{k}} d t
$$

ce qui donne:

$$
P(t) d t=\frac{k}{u_{1}-\varepsilon}\left(\frac{u_{1}-\varepsilon}{t-\varepsilon}\right)^{k+1} d t
$$

et dès lors:

$$
E(S)_{a}=\frac{\mathrm{I}}{k-\mathrm{I}}\left(u_{1}-\varepsilon\right)^{k} \frac{\mathrm{I}}{(L-\varepsilon)^{k-1}}
$$

forme valable pour $k>\mathrm{I}$.

4.3.3. $F(x)$ est du type $I I I$.

En appliquant (3.2I), (4.I) peut s'écrire:

$P(t) d t=\sum_{m=1}^{\infty}\left(\frac{l}{w-u_{1}}\right) \cdot \frac{\mathrm{I}}{(m-\mathrm{I}) !}\left(\frac{w-t}{w-u_{1}}\right)^{l m-1} e^{-\left(\frac{w-t}{w-u_{1}}\right)^{l}} d t \quad(4) \cdot 6$ 
d'où

$$
P(t) d t=\frac{l}{w-u_{1}}\left(\frac{w-t}{w-u_{1}}\right)^{l-1} d t
$$

et dès lors:

$$
E(S)_{a}=\frac{\mathrm{I}}{l+\mathrm{I}}\left(\frac{\mathrm{I}}{w-u_{1}}\right)^{l}(w-L)^{l+1}
$$

\subsection{Conclusions.}

a) Les expressions de $E(S)_{a}$ données par (4.3), (4.5) et (4.7) ont été établies en introduisant dans les calculs les fonctions de densité asymptotiques $T_{m}(x)$.

Comme on a déjà fait remarquer en conclusion du chapitre 3 les expressions asymptotiques de $T_{m}(x)$ ne sont généralement valables que pour $m$ petit par rapport à $n$.

On peut constater que l'expression (4.I) de $P(t) d t$ contient une série de termes pour lesquels $m$ n'est pas petit par rapport à $n$.

Admettre (4.I) comme exact pour le calcul de $E(S)_{a}$ revient à admettre que les propriétés asymptotiques de $F(x)$ sont valables dans tout le domaine $x \geq L$ et dès lors à admettre pour $F(x)$ une fonction bien spécifiée dans le domaine $x \geqslant L$. La méthode employée consiste donc dans le fond à estimer à partir de l'échantillon $\left(x_{1}^{(1)}, x_{1}^{(2)} \ldots x_{1}^{(N)}\right)$ les paramètres de cette fonction.

On démontrera ci-après que les expressions (4.3), (4.5) et (4.7) de $E(S)_{a}$ sont exactes, si $F(x)$ prend pour $x \geqslant L$ respectivement les formes suivantes:

$$
F(x)=\mathrm{I}-\frac{\mathrm{I}}{n} e^{-\alpha_{1}\left(x-u_{1}\right)} \text { et donc } T(x)=\frac{\alpha_{1}}{n} e^{-\alpha_{1}\left(x-u_{1}\right)}
$$

pour le type I

$$
F(x)=\mathrm{I}-\frac{\mathrm{I}}{n}\left(\frac{u_{1}-\varepsilon}{x-\varepsilon}\right)^{k} \text { et } T(x)=\frac{k}{n} \frac{\mathrm{I}}{u_{1}-\varepsilon}\left(\frac{u_{1}-\varepsilon}{x-\varepsilon}\right)^{k+1}
$$

pour le type II

$$
F(x)=\mathrm{I}-\frac{\mathrm{I}}{n}\left(\frac{w-x}{w-u_{1}}\right)^{l} \text { et } T(x)=\frac{l}{n} \frac{\mathrm{I}}{w-u_{1}}\left(\frac{w-x}{w-u_{1}}\right)^{l-1}
$$

pour le type III. 
En effet, la somme $Q(t) d t$ des probabilités que $n$ variables $x_{1}$, $x_{2} \ldots x_{n}$ prennent une valeur comprise entre $t$ et $t+d t$ est donnée par:

$$
Q(t) d t=n T(t) d t
$$

Pour le type I on a en vertu de (4.8):

$Q(t) d t=\alpha_{1} e^{-\alpha_{1}\left(t-u_{1}\right)} d t$ et donc $Q(t) \equiv P(t)$ pour $t \geqslant L$.

Pour le type II on a en vertu de (4.9):

$Q(t) d t=\frac{k}{u_{1}-\varepsilon}\left(\frac{u_{1}-\varepsilon}{t-\varepsilon}\right)^{k+1}$ et donc $Q(t) \equiv P(t)$ pour $t \geqslant L$.

Et enfin pour le type III en vertu de (4.10) on a:

$Q(t) d t=\frac{l}{w-u_{1}}\left(\frac{w-t}{w-u_{1}}\right)^{l-1}$ et donc $Q(t) \equiv P(t)$ pour $t \geqslant L$.

Il s'ensuit que pour les trois types:

$$
E(S)_{a}=\int_{i}^{+\infty}(t-L) Q(t) d t \equiv \int_{\Sigma}^{\infty}(t-L) P(t) d t
$$

et on retrouve donc les expressions (4.3), (4.5) et (4.7), sans faire appel aux distributions asymptotiques $T_{m}(x)$.

b) Compte tenu des hypothèses admises ci-dessus on montrera dans le chapitre 5 que les valeurs calculées de $E(S)_{a}$ sont valables pour une période de temps, pour laquelle on a:

$$
E(i)=n
$$

$E(i)$ étant l'espérance mathématique du nombre de sinistres $i$ survenus pendant la période de temps.

\section{Remarque:}

On peut constater [I] que (4.8), (4.9) et (4.I0) sont les développements de $F(x)$ et $T(x)$ à partir de $u_{1}$, valables asymptotiquement et dont nous acceptons donc la validité dans tout le domaine $x \geqslant L$.

5. THÉORÈME GÉNÉRAL RELATIF AU CALCUL DE E(S)

\subsection{Position du problème.}

a) Pour une période de temps de référence choisie nous disposons

1) E. J. Gumbel: ,Statistics of Extremes" page I62. 
de la loi de survenance des sinistres [I] c'est-à-dire que nous connaissons pour la période de temps choisie les probabilités:

avec

$$
\begin{array}{llllll}
q_{0} & q_{1} & \ldots & q_{i} & \ldots & \text { qu'il survienne } \\
\circ & \mathrm{I} & \ldots & i & \ldots & \text { sinistres } \\
& \multicolumn{5}{c}{\sum_{i=0}^{\infty} q_{i}=\mathrm{I} .}
\end{array}
$$

b) la fonction de répartition $F(x)$ des sinistres survenus $(T(x)$ étant la fonction de densité) n'est pas connue mais demeure inchangée dans le temps.

c) Nous disposons comme au chapitre 4, uniquement de l'échantillon $\left(x_{1}^{(1)}, x_{1}^{(2)} \ldots x_{1}^{(N)}\right)$ extrait de $N$ séries de $n$ sinistres.

Compte tenu de ces hypothèses on se propose de calculer $E(S)$ pour la période de temps de référence choisie ( $S$ étant la somme totale payée par le réassureur pendant cette période).

\subsection{Théorème.}

On a pour la période de temps de référence:

$$
E(S)=E(i) \int_{L}^{\infty}(t-L) T(t) d t
$$

avec $E(i)=\sum_{i=0}^{\infty} i q_{i}$ c.à.d. l'espérance mathématique du nombre de sinistres $i$ survenus pendant la période.

$L$ : l'excess leve].

Cette expression permet le calcul de $E(S)$, soit analytiquement, soit numériquement, dès qu'on dispose d'une "estimation" de $T(x)$ dans le domaine $x \geqslant L$.

\subsubsection{Démonstration au théorème.}

Comme la loi de survenance des sinistres définit une série infinie d'événements contradictoires on peut écrire:

1) ED. Franckx: "Sur la fonction de distribution du sinistre le plus élevé" (.,The Astin Bulletin, Vol. II, Part III, avril 1963, page 4I5). 


$$
E(S)=\sum_{i=0}^{\infty} q_{i} E(S) / i
$$

$E(S) / i$ désignant l'espérance mathématique de $S$ si le nombre de sinistres survenus pendant la période de temps choisie est exactement $i$.

Or:

$$
E(S) / i=i \int_{L}^{+\infty}(t-L) T(t) d t
$$

et dès lors:

$$
E(S)=\left(\sum_{i=0}^{\infty} i q_{i}\right) \int_{L}^{\infty}(t-L) T(t) d t
$$

ce qui démontre le théorème.

\subsubsection{Cas particuliers.}

Si on admet que les expressions (4.8), (4.9) et (4.I0) de $T(x)$ sont valables dans le domaine $x \geqslant L$, alors (5.I) devient:

$$
E(S)=\frac{E(i)}{n} \int_{L}^{\infty}(t-L) \alpha_{1} e^{-\alpha_{1}\left(t-u_{1}\right)} d t
$$

pour le type I.

$$
E(S)=\frac{E(i)}{n} \int_{L}^{\infty}(t-L) \frac{k}{u_{1}-\varepsilon}\left(\frac{u_{1}-\varepsilon}{t-\varepsilon}\right)^{k+1} d t
$$

pour le type II

$$
\text { et } \quad E(S)=\frac{E(i)}{n} \int_{\Sigma}^{\infty}(t-L) \frac{1}{w-u_{1}}\left(\frac{w-t}{w-u_{1}}\right)^{l-1} d t
$$

pour le type III.

Ces trois expressions se réduisent à :

$$
E(S)=\frac{E(i)}{n} E(S)_{a}
$$


$E(S)_{a}$ étant donné par:
(4.3) pour le type I
(4.5) pour le type II
et
(4.7) pour le type III.

a) Si pour la période de temps considérée on a

alors (5.2) devient:

$$
E(i)=n
$$

$$
E(S)=E(S)_{a}
$$

ce qui justifie la remarque faite au 4.4.b.

b) Si la loi de survenance est binomale on a

$$
q_{i}=C_{n}^{i} p^{i}(\mathrm{I}-p)^{n^{\prime}-i}
$$

$$
i=0, \mathrm{x}, \ldots n^{\prime}
$$

alors $E(i)=n^{\prime} p$ et (5.2) devient:

$$
E(S)=\frac{n^{\prime} p}{n} E(S)_{a}
$$

c) Si la loi de survenance est la loi de Poisson de paramètre $\lambda$ on a:

$$
q_{i}=e^{-\lambda} \frac{\lambda^{i}}{i !}
$$

$$
i=0, \mathrm{I} \ldots
$$

alors $E(i)=\lambda$ et $(5.2)$ devient:

\subsection{Conclusions.}

$$
E(S)=\frac{\lambda}{n} E(S)_{a}
$$

Si l'expression (5.2) donne une valeur $E(S)$, qui peut théoriquement servir comme valeur de base pour le calcul d'une prime de réassurance, il est toutefois évident que l'application pratique de cette formule nécessite encore:

a) la mise au point de procédés pratiques permettant l'estimation des paramètres intervenant dans l'expression de $E(S)$.

b) l'étude des corrections à apporter dans le cas où le matériel statistique disponible ne serait pas exactement celui employé dans le modèle théorique étudié.

Je tiens à remercier Monsieur le Professeur Ed. Franckx de l'Ecole Royale Militaire et Monsieur P. Gennart, chargé de cours à l'Ecole Royale Militaire, pour leurs précieux conseils. 